\title{
Образование дефектов в структурах GaAs c непокрытой и покрытой пленкой AIN поверхностями при имплантации ионов азота и последующем отжиге
}

\author{
(ㄱ Н.А. Соболев, В.И. Сахаров, И.Т. Серенков, А.Д. Бондарев, К.В. Карабешкин, \\ Е.В. Фомин, А.Е. Калядин, В.М. Микушкин, Е.И. Шек, Е.В. Шерстнев
}

Физико-технический институт им. А.Ф. Иоффе Российской академии наук, 194021 Санкт-Петербург, Россия

E-mail: nick@sobolev.ioffe.rssi.ru

(Поступила в Редакцию 6 декабря 2018 г.

В окончательной редакции 9 декабря 2018 г.

Принята к публикации 12 декабря 2018 г.)

\begin{abstract}
Исследованы профили концентрации дефектов, образовавшихся в структурах после имплантации ионов азота в эпитаксиальные слои GaAs c непокрытой и покрытой пленкой AlN поверхностями и последующего отжига. Энергии ионов и дозы имплантации выбирались таким образом, чтобы концентрационные профили атомов азота совпадали в структурах обоих типов. В исследуемых образцах измерялись спектры обратного резерфордовского рассеяния протонов в случайном и каналирующих режимах и рассчитывались профили концентрации образовавшихся точечных дефектов. Установлено, что имплантация ионов азота вводит примерно одинаковое количество точечных дефектов в оба типа структур, а формирование пленки AlN с помощью ионно-плазменного распыления сопровождается образованием дополнительного количества дефектов. Однако после отжига структур обоих типов концентрация остающихся дефектов примерно одинакова.
\end{abstract}

DOI: 10.21883/FTP.2019.04.47435.9038

\section{1. Введение}

Для создания эффективных источников излучения в инфракрасной области спектра проводятся обширные исследования разбавленных твердых растворов $\mathrm{GaAs}_{1-x} \mathrm{~N}_{x}$ [1-12]. Для получения этих растворов используются различные технологические методы, в том числе металлорганическое химическое напыление [1], газофазная эпитаксия из металлоорганического источника при атмосферном давлении [2], различные модификации молекулярно-пучковой эпитаксии [3-6] и ионная имплантация [7-12]. Ионная имплантация является простым и эффективным методом введения азота в GaAs c концентрацией выше равновесной, но при этом важную роль в решении задачи получения твердых растворов приборного качества играет контроль вводимых имплантационных дефектов и методы их устранения $[7,8,10]$. В опубликованных статьях содержится очень мало такой информации. Недавно нами были представлены результаты исследования методом обратного резерфордовского рассеяния дефектов в слоях $\mathrm{GaAs}$ : N, образующихся при имплантации ионов азота с одной энергией и разными дозами $[11,12]$. Для создания эффективных светоизлучающих структур $\mathrm{GaAs}_{1-x} \mathrm{~N}_{x}$ необходимо иметь достаточно толстый слой твердого раствора с высокой и постоянной концентрацией атомов азота $[\mathrm{N}]$. После имплантации ионов азота для устранения введенных радиационных нарушений и формирования слоя GaAsN проводится термический отжиг. В отличие от кремния, при отжиге имплантированных слоев GaAs происходит диссоциация поверхностных слоев за счет потери ато- мов мышьяка. Поэтому при отжиге необходимо нанести на поверхность защитный слой или проводить отжиг в соответствующей контролируемой атмосфере. Одним из таких защитных покрытий может служить слой нитрида алюминия AlN. Bo-первых, в отличие из защитных слоев $\mathrm{SiO}_{2}$ и $\mathrm{Si}_{3} \mathrm{~N}_{4}$, он препятствует диффузии атомов галлия наружу. Во-вторых, поскольку коэффициенты термического расширения AlN и GaAs достаточно близки, во время отжига практически не возникает дополнительных напряжений, а также сохраняются хорошие адгезионные свойства. Насколько нам известно, исследований дефектов, возникающих во время формирования такого защитного слоя, не проводилось. В настоящей статье приводятся результаты исследования процессов образования дефектов при нанесении защитного слоя AlN на эпитаксиальный слой GaAs и имплантации ионов азота с несколькими энергиями в структуры AlN/GaAs, а также их отжига во время последующей термообработки.

\section{2. Экспериментальные условия}

В качестве исходных образцов использовались сильно легированные (100) GaAs подложки, на которых методом газофазной эпитаксии (ГФЭ) в хлоридной системе выращивались нелегированные слои GaAs n-типа проводимости с толщиной $\sim 40$ мкм. На части образцов методом реактивного ионно-плазменного распыления наносились пленки AlN толщиной 70 нм [13]. Имплантация ионов $\mathrm{N}^{+}$проводилась при комнатной температуре и плотности тока $0.25 \mathrm{M \kappa} / \mathrm{cm}^{2}$ на ускорителе фирмы 
High Voltage Engineering Europe в образцы без и с защитной пленкой $\mathrm{AlN}$ ( $\mathrm{GaAs}$ и $\mathrm{Al} / \mathrm{GaAs}$ ). В нашей предыдущей работе [11] было установлено, что рассчитанные по программе TRIM [14] концентрационные профили атомов азота $([\mathrm{N}])$ в имплантированном слое $\mathrm{GaAs}$ достаточно точно описывают экспериментальные профили, измеренные с помощью метода вторичной ионной масс-спектрометрии (SIMS). Поэтому расчет концентрационного профиля в данной работе также проводился с использованием программы TRIM. Для того чтобы в структурах с непокрытой и покрытой пленкой $\mathrm{AlN}$ поверхностями профили введенных при имплантации ионов азота совпадали, использовались следующие энергии (кэВ) и дозы $\left(\mathrm{cm}^{-2}\right): 250 / 10 \cdot 10^{15}+$ $+140 / 4.5 \cdot 10^{15}+70 / 3.3 \cdot 10^{15}+30 / 1.3 \cdot 10^{15}$ (для GaAs : N) и $310 / 10.6 \cdot 10^{15}+190 / 3.7 \cdot 10^{15}+130 / 3.2 \cdot 10^{15}$ $+80 / 2.2 \cdot 10^{15}$ (для Al/GaAs: N). При выбранных значениях энергий и доз имплантации в диапазоне глубин 120-490нм концентрация атомов азота равнялась $3.3 \cdot 10^{20} \mathrm{~cm}^{-3}$. Отжиги проводились при температуре $700^{\circ} \mathrm{C}$ в течение 60 мин в потоке $\mathrm{Ar}$.

Метод обратного резерфордовского рассеяния (RBS) протонов с энергией 227 кэВ использовался для исследования дефектной структуры имплантированных слоев. Измерение RBS спектров обратно рассеянных протонов проводилось в режимах каналирования и псевдослучайного направления пучка (далее каналирующий и случайный режимы). Кремниевые детекторы с энергетическим разрешением 3.5 кэВ регистрировали ионы, рассеянные на угол $170^{\circ}$. Расчет профилей концентрации введенных точечных дефектов проводился с помощью процедуры, предложенной в [15]. Для расчета профиля введенных дефектов в системе AlN/GaAs: N была разработана программа, в которой были учтены потери энергии ионов при прохождении покрывающего слоя, а также деканалирование при рассеянии ионов на атомах слоя AlN.

\section{3. Экспериментальные результаты и обсуждение}

Спектры обратного рассеяния протонов в образцах без пленки AlN после имплантации ионов азота и последующего отжига, измеренные в случайном и каналирующем режимах, приведены на рис. 1 (кривые 1-3). Анализ спектров показывает, что аморфизации имплантированного слоя не происходит, поскольку интенсивность случайного сигнала во всем диапазоне измерений выше, чем у каналирующего. На рисунке также приведен каналирующий спектр для исходного образца перед имплантацией (кривая 4). Хорошо видно, что постимплантационный отжиг не восстанавливает дефектную структуру образца до уровня в исходном образце. Профили концентрации точечных дефектов, рассчитанные из RBS спектров и нормированные на число атомов в $1 \mathrm{~cm}^{-3}$, для образцов GaAs : $\mathrm{N}$ после имплантации и последующего отжига приведены на рис. 2 (кривые 1 и 2 соответственно). После имплантации во всем диапазоне глубин с одинаковой концентрацией атомов азота наблюдается заметное уменьшение концентрации введенных дефектов по направлению к поверхности (кривая 1). Возможно, наличие градиента обусловлено диффузией точечных дефектов к поверхности во время имплантации. Максимальный уровень радиационных нарушений достигает 30\%. Высокая концентрация введенных дефектов около поверхности после имплантации обусловлена образованием тонкого $\sim 10$ нм сильно дефектного слоя. Концентрация дефектов в этой области существенно уменьшается после отжига. Следует отметить, что концентрация образовавшихся при имплантации дефектов существенно меньше, чем рассчитанная с помощью программы TRIM. Этот эффект ранее наблюдался в случае имплантации легких ионов в матрицу, состоящую из тяжелых ионов, и, в частности, для системы GaAs : N [11].

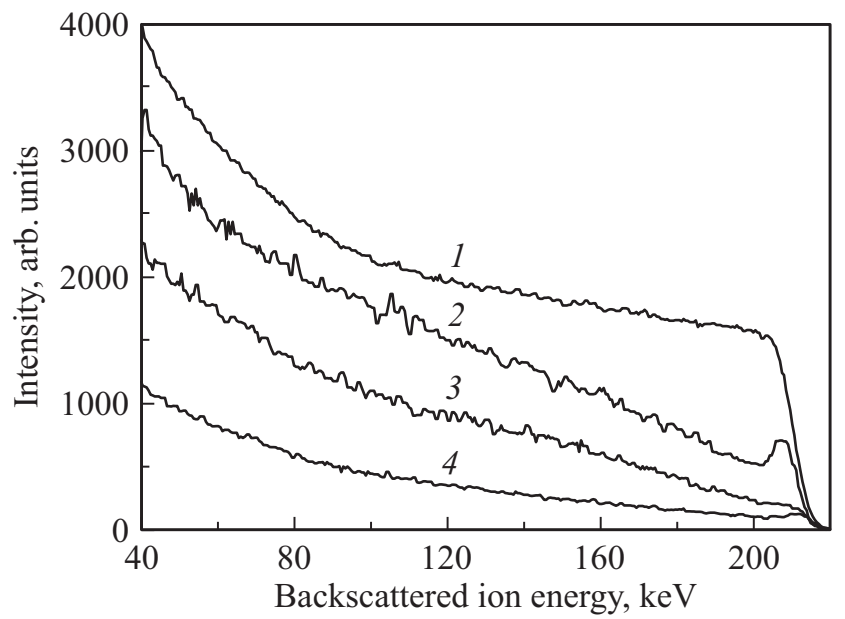

Рис. 1. Спектры обратного резерфордовского рассеяния протонов в имплантированных структурах GaAs: N: случайный (1) и каналированные в образцах после имплантации ионов азота (2), последующего отжига (3) и перед имплантацией (4).

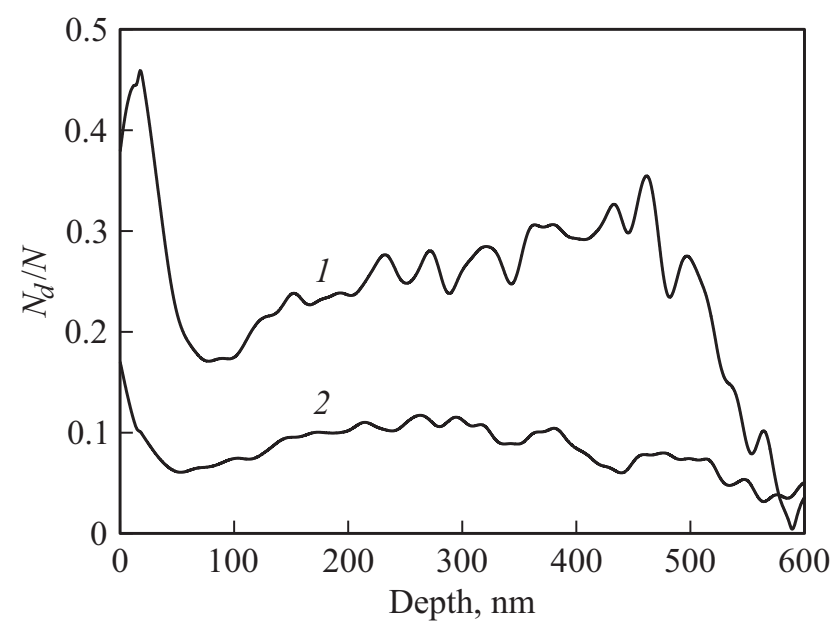

Рис. 2. Концентрационные профили дефектов, образовавшихся в структурах GaAs : N, после имплантации (1) и последующего отжига (2). 


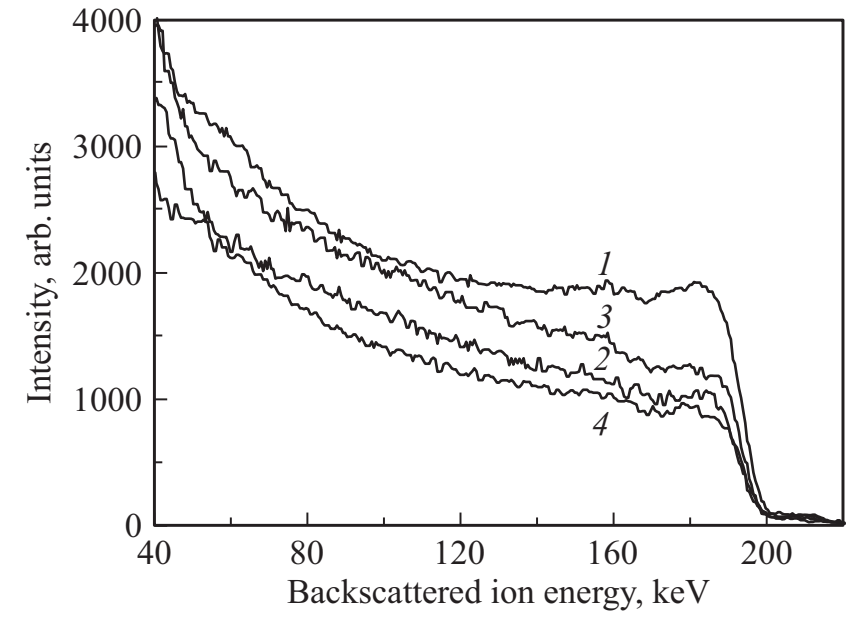

Рис. 3. Спектры обратного резерфордовского рассеяния протонов в имплантированных структурах AlN/GaAs : N: случайный (1) и каналированные в образцах после нанесения слоя AIN (2), имплантации ионов азота (3) и последующего отжига (4).

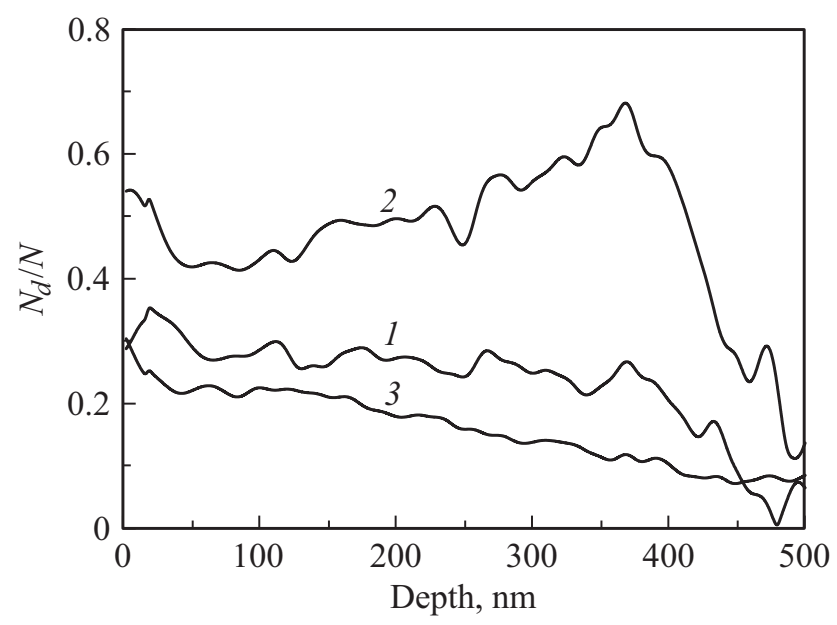

Рис. 4. Концентрационные профили дефектов, образовавшихся в структурах AlN/GaAs : N, после нанесения слоя $\operatorname{AlN}(1)$, имплантации ионов азота (2) и последующего отжига (3).

Отжиг образца снижает концентрацию образовавшихся во время имплантации дефектов более чем в 2 раза. При этом наиболее эффективно удаляются дефекты из области их максимальной концентрации. Полностью удалить дефекты в процессе отжига не удалось.

Спектры обратного рассеяния протонов в образцах, покрытых пленкой AlN после имплантации ионов азота и последующего отжига, измеренные в случайном и каналирующем режимах, приведены на рис. 3 (кривые $1,3,4)$. Как и в случае образцов без пленки AlN, аморфизации имплантированного слоя не происходит. На рисунке также приведен каналирующий спектр для образца после нанесения пленки (кривая 2). Хорошо видно, что постимплантационный отжиг улучшает дефектную структуру имплантированного образца по срав- нению даже с образцом перед имплантацией. Нормированные профили концентрации точечных дефектов, рассчитанные из RBS спектров, для образцов AlN/GaAs : N после различных воздействий приведены на рис. 4 (кривые $1-3$ ). Глубины для образцов с пленкой AlN отсчитываются от интерфейса AlN/GaAs. Уменьшение концентрации точечных дефектов с глубиной (кривая 1) свидетельствует о том, что в процессе формирования пленки AlN источником их образования является поверхность. Это хорошо согласуется с известным фактом, что в процессе ионно-плазменного распыления образуется достаточно высокая концентрация неравновесных точечных дефектов. Максимальный уровень радиационных нарушений после имплантации достигает $62 \%$. Сравнение концентрационных профилей дефектов после имплантации в структуры с непокрытой и покрытой пленкой нитрида алюминия поверхностями (кривая 1 на рис. 2 и кривая 2 на рис. 4 ) свидетельствует о том, что вводится примерно одинаковое количество дефектов, как и следовало ожидать для выбранных режимов имплантации. Постимплантационный отжиг достаточно эффективно уменьшает концентрацию дефектов и в структурах с покрытой поверхностью: их концентрация даже ниже, чем в структурах после формирования пленки AlN (cp. кривую 2 на рис. 2 и кривую 3 на рис. 4). Следует отметить, что отжиг более эффективно снижает концентрацию введенных при имплантации дефектов, чем образовавшихся дефектов при плазменной обработке. Поэтому необходимо проведение дополнительных исследований по совершенствованию процесса формирования пленок AlN.

\section{4. Заключение}

Имплантация ионов азота в эпитаксиальный слой GaAs-структур c непокрытой и покрытой пленкой AlN поверхностями c четырьмя энергиями в диапазоне $30-310$ кэВ и парциальными дозами $1.3 \cdot 10^{15}-1 \cdot 10^{16} \mathrm{~cm}^{-2}$ не приводит к аморфизации этого слоя. Формирование пленки нитрида алюминия с помощью метода реактивного ионно-плазменного распыления приводит к введению в приповерхностную область GaAs радиационных нарушений на уровне $25 \%$. Имплантация ионов азота вводит примерно одинаковое количество точечных дефектов в оба типа структур. Последующий отжиг при температуре $700^{\circ} \mathrm{C}$ в течение 60 мин в потоке $\mathrm{Ar}$ снижает концентрацию радиационных дефектов более чем в 2.5 раза. Наиболее эффективно удаляются дефекты из области максимальной концентрации введенных атомов азота. Важно, что в отожженных образцах с пленкой нитрида алюминия концентрация остающихся дефектов ниже, чем в этих образцах перед имплантацией.

Авторы выражают благодарность В.Н. Пантелееву за помощь в проведении отжига имплантированных слоев. 
Работа поддержана Российским научным фондом (проект № 17-19-01200).

\section{Список литературы}

[1] M. Weyers, M. Sato, H. Ando. Jpn. J. Appl. Phys., pt 2, 31, L853 (1992).

[2] A. Ougazzaden, Y.Le Bellego, E.V.K. Rao, M. Juhel, L. Leprince, G. Patriarche. Appl. Phys. Lett., 70, 2861 (1997).

[3] M. Kondow, K. Uomi, K. Hosomi, T. Mozume. Jpn. J. Appl. Phys., pt 2, 33, L1056 (1994).

[4] K. Uesugi, N. Morooka. Appl. Phys. Lett., 74, 1254 (1999).

[5] А.Е. Жуков, Е.С. Семенова, М.В. Устинов, E.R. Weber. ЖТФ, 71 (10), 59 (2001).

[6] А.Ю. Егоров, Е.С. Семенова, В.М. Устинов, Y.G. Hong, C. Tu. ФТП, 36 (9), 1056 (2002).

[7] K.M. Yu, W. Walukiewicz, J. Wu, J.W. Beeman, J.W. Ager III, E.E. Haller, W. Shan, H.P. Xin, C.W. Tu, C. Ridgway. J. Appl. Phys., 90, 2227 (2001).

[8] X. Weng, S.J. Clarke, W. Ye, S. Kumar, R.S. Goldmana, A. Daniel, R. Clarke, J. Holt, J. Sipowska, A. Francis, V. Rotberg. J. Appl. Phys., 92, 4012 (2002).

[9] V.M. Mikoushkin. Appl. Surf. Sci., 257 (11), 4941 (2011).

[10] Kun Gao, S. Prucnal, W. Skorupa, M. Helm, S. Zhou. Appl. Phys. Lett., 105, 012107 (2014).

[11] Н.А. Соболев, Б.Я. Бер, Д.Ю. Казанцев, А.Е. Калядин, К.В. Карабешкин, В.М. Микушкин, В.И. Сахаров, И.Т. Серенков, Е.И. Шек, Е.В. Шерстнев, Н.М. Шмидт. Письма ЖТФ, 44 (13), 44 (2018).

[12] Н.А. Соболев, А.Е. Калядин, К.В. Карабешкин, Р.Н. Кютт, В.М. Микушкин, Е.И. Шек, Е.В. Шерстнев, В.И. Вдовин. Письма ЖТФ, 44 (18), 24 (2018).

[13] Н.А. Берт, А.Д. Бондарев, В.В. Золотарев, Д.А. Кириленко, Я.В. Лубянский, А.В. Лютецкий, С.О. Слипченко, А.Н. Петрунов, Н.А. Пихтин, К.Р. Аюшева, И.Н. Арсентьев, И.С. Тарасов. ФТП, 49, 1429 (2015).

[14] J.F. Ziegler, J.P. Biersack, U. Littmark. The Stopping and Range of Ions in Solids (Pergamon Press, N.Y., 1985).

[15] L.C. Feldman, J.W. Mayer, S.T. Picraux. Material Analysis by Ion Channeling (Academic Press, N.Y., 1982) chap. 5.

\section{Defect Formation in GaAs structures with Surfaces Noncovered and Covered by an AIN Film at Nitrogen Ion Implantation and Subsequent Annealing}

\author{
N.A. Sobolev, V.I. Sakharov, I.T. Serenkov, \\ A.D. Bondarev, K.V. Karabeshkin, E.V. Fomin, \\ A.E. Kalyadin, V.M. Mikoushkin, E.I. Shek, \\ E.V. Sherstnev \\ loffe Institute, \\ 194021 St. Petersburg, Russia
}

\begin{abstract}
Concentration profiles of defects produced in structures after nitrogen ion implantation into GaAs epitaxial layers with surfaces bare and covered by an AlN film and subsequent annealing were studied. Ion energies and implantation doses were chosen so that the nitrogen atom concentration profiles coincide in the structures of both types. Spectra of Rutherford backscattering of protons were measured in random and channeling regimes and concentration profiles of produced point defects were calculated in studied samples. It was determined that nitrogen ion implantation produces practically the same quantity of the point defects within the structures of both types and coating the AlN film by ion-plasma sputtering is followed by formation of additional defects. However, annealing of the structures of both types leads to practically the same concentration of residual defects.
\end{abstract}

